\title{
Analysis of Turbidity Measurement Based on Ratio Method
}

\author{
Nan Xie ${ }^{1, a^{*}}$, Weimin Chen ${ }^{2, b}$, Zhennan Zhang ${ }^{2, c}$ and Yijia Yuan ${ }^{1, d}$ \\ ${ }^{1}$ Zhejiang University of Water Resource and Electric Power, Hangzhou, China \\ ${ }^{2}$ College of Mechanical and Electrical Engineering, China Jiliang University,Hangzhou, China \\ a595075251@qq.com, ${ }^{\text {b }}$ C9419@sina.com, ${ }^{c}$ zhangzn@sina.com, ${ }^{\text {d } 905321889 @ q q . c o m ~}$
}

Keywords: Turbidity measurement; Ratio method; Signal processing; Circuit design

\begin{abstract}
Fine suspended matter such as sediment, organic matter and inorganic matter can absorb a large amount of bacteria, viruses and other harmful substances, which is a threat to human health, and water turbidity is one of the important indicators of water quality monitoring. The ratio method of the photoelectric detection about turbidity measurement has been applied, and the corresponding hardware circuit design and software design of the turbidity measurement system also have been done. The water turbidity measurement system can complete data acquisition and data analysis, and compare with the relation curves between the data measured by the system's turbidity sensor and the actual turbidity value, and the least square method is used for modeling analysis and data compensation. The experimental results show that the ratio method is more accurate than the transmission method and scattering method to measure the turbidity of water samples, and the method has better interference rejection. The system design can be used in different water turbidity measurement or correction, and it has good market application prospect.
\end{abstract}

\section{Introduction}

With the rapid growth of China's economy, the phenomenon of water pollution is becoming more and more serious, containing a large number of harmful chemical substances of pollutants discharged into natural water, these pollutants not only have caused the serious environmental pollution, but also restricts the development of society and economy. No matter the research institutions or the environmental protection departments focus on water turbidity detection, because the water supply network water turbidity control not only affects the safe of people water supply, industrial production, but also the product quality and operation process. How to deal with water management has already become one of issues of our country, because the water quality is closely related to people's life health and industrial production. Turbidity detection is very important in the people daily life or in industrial production. In the turbidity detection, we will encounter a variety of organic impurities, color, light, air, temperature and electrical interference factors, so in detection modeling we must take account on the influence brought by these interference factors, and down to a minimum.

In 2013, Zhejiang Province has produced five water treatment policy, which is sewage treatment, flooding prevention, drainage water, supplying water and water conservation. And the key problem of sewage treatment and supplying water is water quality, and turbidity is one of important indexes to evaluate the water quality. In view of the current background combined with the latest water quality turbidity detection technology, computer technology and in the existing turbidity sensor for measurement of turbidity water quality analysis, the authors do some comparison of three kinds of photoelectric detecting turbidity measurement method, and select the ratio method turbidity measurement, and design the corresponding system circuits. Last the authors have done experimental determination and analysis, used least square method for data modeling and calculation [1-2] to obtain the optimal turbidity value, and make the relative error minimized. 


\section{Turbidity Measurement Method}

Water quality turbidity detection is mainly considered the light intensity measurement, the methods mainly include transmission method, scattering method and the scattered light and transmitted light ratio method and so on [1,3-5].

Transmission Measurement Method. Transmission measurement method follows the Lambert Beer law, which refers a beam of collimated monochromatic light along the projected in a direction in a homogeneous medium, light intensity attenuation amount and intensity and the thickness of the medium is proportional to the product, so by measuring the tested sample solution of intensity of light, the water turbidity value would be calculated. The linear element is used for photoelectric conversion, and the signal follows Eq. (1).

$$
\mathrm{I}_{\mathrm{t}}=\mathrm{AI}_{0} \mathrm{e}^{-\mathrm{KTL}}
$$

Where, $I_{t}$ is transmitting light intensity, A is constant circuit, $I_{0}$ is the intensity of incident light, $\mathrm{K}$ is constants associated with incident light wavelength and particle distribution, $\mathrm{T}$ is turbidity of measured water sample, $\mathrm{L}$ is transmission path.

Fig. 1 (a) is the output characteristic curve of the sample liquid using the transmission measurement method, it shows that the photoelectric conversion signal and the turbidity is a logarithmic relationship, and the I_t and T keep the monotonous attenuation relationship. In the actual situation, transmission method satisfies the linear relationship only in a certain range. But in low turbidity, the turbidity changes caused unobvious change of the transmission light intensity, then detection accuracy would be low and the measurement error has been increased. When the turbidity is large, the transmission intensity and the turbidity of the light show a nonlinear relationship, then the transmission light would be weak and measurement error would be large, so the transmission measurement method is suitable for testing the high turbidity water sample.

Scattering Measurement Method. In the incident light meeting the water particles will cause the scattering light. The intensity of scattered light is related to the number and diameter of the water sample particles, so we can obtain the turbidity information by measuring the scattered light intensity [2-4]. When the particle in water is much smaller than the incident wavelength, the volume of water produces 90。 scattering light intensity, which obeys the Rayleigh Law shown as Eq. (2).

$\mathrm{I}_{\mathrm{s}}=\mathrm{BTI}_{0}$

Where, $\mathrm{B}$ is the ratio of the incident light wavelength, refractive index, etc. When the particle in water is close to the incident wavelength, the volume of water produces 90 。 scattering light intensity, which obeys the Mie Law shown as Eq. (3).

$\mathrm{I}_{\mathrm{s}}=\mathrm{CTI}_{0}$

Where, $\mathrm{C}$ is the ratio of Michaelis coefficient, particle light interception area etc.

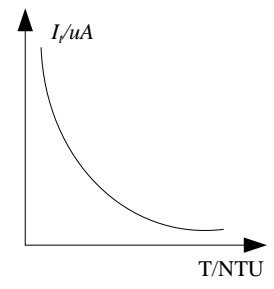

(a)

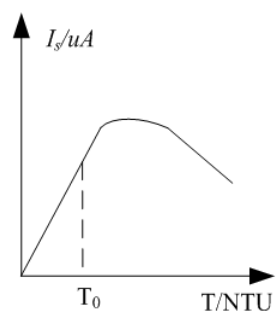

(b)

Figure 1. Output characteristic curve of transmission measurement $\&$ scattering method

The scattered light measurement method can be carried out according to different angles, mainly be divided into the forward light measurement, 90。 scattering measurement and post - optical scattering measurement. However, 90。 Measurement is the most stable and the most common international method. The measurement of turbidity by scattering method is a reference to ISO7027, and Fig. 1 (b) is the output characteristic curve using 90。 scattering measurement. Seen from the Fig. 1 (b), when the sample liquid turbidity is low, the turbidity value and the light scattering intensity is 
linear relationship; but with increasing turbidity, the relationship is nonlinear [1, 6-7] , also, it brings large errors to turbidity measurements.

Ratio Measuring Method. When light passes through water samples, it produces some transmission light and scattering light, and scattered light would become attenuate before reaching the photoelectric receiver, just as Eq. (4) said.

$$
\mathrm{I}_{\mathrm{s}}=\mathrm{K}_{\mathrm{s}} \mathrm{I}_{0} \mathrm{Te}^{-\mathrm{kTL}}
$$

Where, $\mathrm{K}_{\mathrm{s}}$ is a ratio coefficient. If the transmission path is equal to the scattering path, we can obtain Eq. (5) by Eq. (1) and Eq. (4).

$$
\frac{\mathrm{I}_{\mathrm{S}}}{\mathrm{I}_{\mathrm{t}}}=\frac{\mathrm{K}_{\mathrm{S}} \mathrm{I}_{0} \mathrm{Te}}{\mathrm{AI}_{\mathrm{o}} \mathrm{e}^{-\mathrm{kTTL}}}=\frac{\mathrm{K}_{\mathrm{S}} \mathrm{T}}{\mathrm{A}}=\mathrm{DT}
$$

Where, $\mathrm{D}$ is a ratio constant. The ratio measurement method is to calculate the ratio of scattered light and transmitted light intensity and then get the turbidity of water sample. And the measurement principle is shown as Fig. 2. When we use the ratio measuring, we find the the linear relationship between the turbidity of water sample and the light intensity.

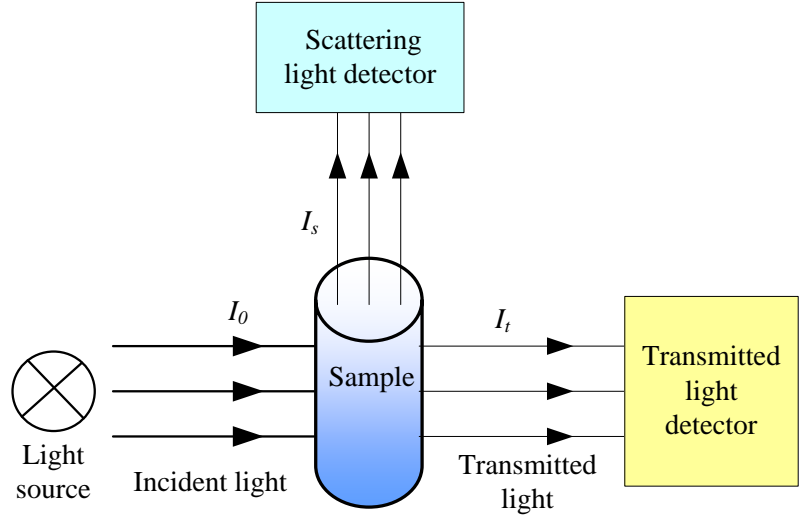

Figure 2. Ratio turbidity measuring principle

\section{Circuits Design of the Measurement System}

Measurement System Design. The same incident light will produce different scattering intensity and the transmission light intensity by different turbidity of measured liquids. We can collect these lights' intensity by photoelectric sensor, and convert the received the optical signal into electrical signal, then amplify and filter by instrument amplifier or filter. After A/D sampling, the deposed signals would send the STM32 microprocessor for signal processing and display [6-10], and achieve detecting the turbidity of the various water[4-7].

Circuits Design of Photoelectric Receiving Signal Processing. The photoelectric receiving diode realizes the conversion of the light signal into a current signal. In signal detection process, the photoelectric diode received current signal is small and it may be low to $\mu \mathrm{A}$ and contains noise. Therefore, the receiving signal processing circuits are very important to the measurement system, which mainly include signal amplifying circuit and filtering circuit and the background light elimination circuit [7-9].

Amplifying and Filtering Circuit Design. The primary amplifier circuit and filter circuit of the photodiode receiving circuit is shown as Fig. 3. After receiving the detection light intensity, the output pulse current $\mathrm{i}(\mathrm{t})$ flows through the R10 and operational amplifier A2, and the output voltage is shown as Eq. (6).

$$
\mathrm{u}_{1}(\mathrm{t})=-i(\mathrm{t}) \times \mathrm{R}_{10}
$$

Considering self-excited oscillation, the capacitor $\mathrm{C} 2$ is connected in parallel on the branch of the feedback resistor R10 series. As shown in Fig. 3, if the value of R10 is bigger, the output voltage can obtain higher value. Meanwhile, it will bring great noise, so the amplifier should maintain a maximum output voltage, which is below $0.5 \mathrm{~V}$. 


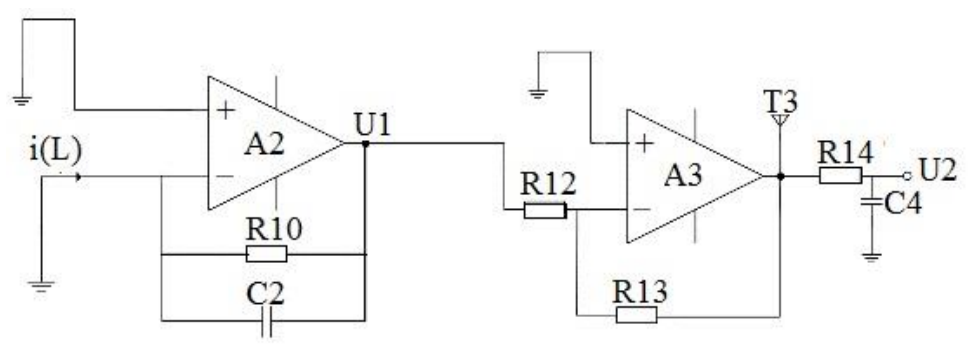

Figure 3. Circuit design of signal amplifying and filtering

Circuit Design of Background Light Noise Cancellation. In the application of photoelectric sensor, eliminating the influence of background light on the detection results is an important means to improve the detection accuracy. In this paper, two sampling and holding circuit and related control measures are used to eliminate the background light. The circuit includes two electronic switches and two sample holders constituted capacitances and resistances.

\section{Experiment and Result Analysis}

Due to the different standard solution and methods of measurement, the experiments have been done in accordance with the provisions of the international standard ISO7027 water turbidity measurement by the international general turbidity standard solution preparation method (formazine) and turbidity units (NTU).

We will put 400NTU formazine standard solution into zero turbidity water for gaining 9 kinds of formazine solution for turbidity measurement , such as $0,10,20,50,100,150,200,250,300 \mathrm{NTU}$. Each formazine solution will complete 3 times measuring, and the measurement results have been shown as Table 1.

Table 1 Result of Formazine solution measurement

\begin{tabular}{|c|c|c|c|c|c|}
\hline \multicolumn{2}{|c|}{ Measured value[NTU] } & Average \\
No.1 & No.2 & No.3 & $\begin{array}{c}\text { Standard turbidity } \\
{[\text { NTU }]}\end{array}$ & $\begin{array}{c}\text { Error } \\
{[\text { NTU] }}\end{array}$ \\
\hline 1.3 & 1.0 & 1.1 & 1.1 & 0.0 & 1.1 \\
\hline 10.2 & 10.3 & 9.9 & 10.1 & 10.0 & 0.1 \\
\hline 19.9 & 20.8 & 20.4 & 20.4 & 20.0 & 0.4 \\
\hline 50.7 & 49.8 & 50.4 & 50.3 & 50.0 & 0.3 \\
\hline 99.2 & 100.3 & 100.8 & 100.1 & 100.0 & 0.1 \\
\hline 150.9 & 151.0 & 149.8 & 149.7 & 150.0 & -0.3 \\
\hline 200.8 & 201.2 & 200.5 & 200.5 & 200.0 & 0.5 \\
\hline 250.8 & 251.1 & 249.9 & 250.6 & 250.0 & 0.6 \\
\hline 300.4 & 299.4 & 299.3 & 299.7 & 300.0 & -0.3 \\
\hline
\end{tabular}

The measurement data shows that the linear error of the measurement system is $\pm 3 \%$, and the least square method is used to compensate the measurement data, which makes the data more accurate in the actual turbidity test.

\section{Conclusion}

Experimental results show that the ratio method of water turbidity measurement system design can eliminate water color, light environment and other external factors. And it is more anti-jamming capability than transmission method or scattering method to measuring water turbidity. Also, in low turbidity measurement, the photoelectric sensors, peripheral circuits, signal processing are easy to realize when we use ratio measurement method, and we could also reduce hardware cost. The measurement system has good market application and popularization value. 


\section{Acknowledgements}

This work was supported by Zhejiang Key Discipline of Instrument Science \& Technology and the 2015 New Talents Project of Zhejiang Province (Project CODE: 2015R434009).

\section{References}

[1] Ji Yinglei. The Research and Design of Intelligent Turbidity Sensor [D]. University of Science and Technology of China, 2014.05.

[2] Luo Yonggang, Cheng Hongyu, Zou Jun, etc. Design of a scattering turbidity sensor [J]. Transducer and Microsystem Technologies, 2015(06): 67-69.

[3] Kang Ya, Zhu Guangping. Discussion on the measurement of turbidity by ratio method [J]. Electronic Technology \& Software Engineering, 2013(21):135-136

[4] Shu Shihu. Water Distribution System Model Automatic Calibration Based On Fmga And Its Applications [D]. Harbin Institute of Technology, 2009.06.

[5] LAN Yu, Zhang Shunxing. Design of photoelectric turbidity measurement system based on AT89C51 [J]. Information Technology, 2013(06): 41-43.

[6] Zeng Zhengren, Yu Jian, Shen Shiquan, etc. Study on the variation law of water turbidity in the main body of the simulated pipe network system [J]. City and town water supply, 2014(02): 68-70.

[7] Song Liping. Application and improvement of 1720 E turbidity meter in wastewater monitoring. Egang Science \& Technology, 2014(01): 41-42.

[8] Chen Dan, Chen Jian. Design of scattering infrared turbidity sensor [J]. Microcomputer Information, 2009(19): 97-98.

[9] Wu Gang, Liu Yueming, Gao Xiaoliang, etc. Design of Dual-channel Turbidity Sensor by Using Optical Fibers [J]. Instrument Technique and Sensor, 2014(10): 14-16.

[10] STM32. http://www.stmcu.com.cn/mcu.html 